\title{
VARIAÇÃO DO TEOR DE UMIDADE E DA DENSIDADE BÁSICA NA MADEIRA DE SETE ESPÉCIES DE EUCALIPTO ${ }^{1}$
}

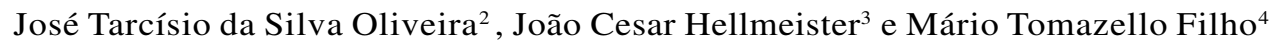

\begin{abstract}
RESUMO - As variações de umidade e da densidade do lenho das árvores são as principais causas dos defeitos de secagem, como o empenamento e fendilhamento das peças de madeira. Os tipos de madeira presentes em um tronco estão relacionados com as variações dessas duas importantes propriedades físicas. Os gradientes de umidade e da densidade da madeira de sete espécies de eucalipto foram avaliados nas direções radial e longitudinal do tronco de árvores recém-abatidas. Os resultados apontaram uma maior homogeneidade de distribuição de umidade dentro das árvores de E. paniculata e E. citriodora, indicada pelos coeficientes de variação e desviopadrão. O diferencial de umidade da madeira nas regiões internas do tronco de E. paniculata e E. citriodora foi de $20 \%$ e de $E$. urophylla e E. grandis, de $80 \%$. A densidade básica da madeira aumentou na direção radial do tronco, e cada espécie de eucalipto apresentou um modelo de variação.
\end{abstract}

Palavras-chave: Madeira, eucalipto, teor de umidade e densidade básica.

\section{VARIATION OF THE MOISTURE CONTENT AND SPECIFIC GRAVITY IN THE WOOD OF SEVEN EUCALYPT SPECIES}

\begin{abstract}
The occurrence of high moisture and density gradients inside of the tree is related with drying defects, especially those characterized by warp and split of the wood. The unequal behavior of wood pieces due to variation of these two properties can be attributed to differences within the wood that constitutes the log. Considering the importance of knowing the moisture and density gradients in eucalyptus trees, this work had as objective evaluating the variations of the moisture content and specific gravity in the radial and longitudinal directions of the trees of seven eucalypts species. Based on the results concerning the moisture distribution inside the tree, there was a great homogeneity in the species of $\boldsymbol{E}$. paniculata and $\boldsymbol{E}$. citriodora, sustained by the low values of variation coefficient and standard deviation. In these species, the moisture differential among the inner and outer parts of the log rarely reached $20 \%$, while for the logs of $\boldsymbol{E}$. urophylla and $\boldsymbol{E}$. grandis, this diferencial reached $80 \%$. In spite of the specific gravity general increased in the direction pithto-bark, each species presented a specific profile of variation, also with variants for each height of the log.
\end{abstract}

Key words: Eucalypt wood, moisture content and specific gravity.

\footnotetext{
${ }^{1}$ Recebido em 31.03.2003 e aceito para publicação em 25.11.2004.

${ }^{2}$ Departamento de Engenharia Rural da UFES. Alegre, ES.

${ }^{3}$ Laboratório de Madeiras e Estruturas de Madeiras, EESC/USP. São Carlos, SP (Falecido em 1999).

${ }^{4}$ Departamento de Ciências Florestais, ESALQ/USP. Piracicaba, SP.
} 


\section{INTRODUÇÃO}

A determinação do teor de umidade e de sua variação no tronco das árvores ou das peças de madeira é de extrema importância no seu desempenho e utilização. Os elevados gradientes de umidade da madeira constituem-se em uma das causas de defeitos de secagem, notadamente os caracterizados por empenamentos e fendilhamentos. As informações relativas à distribuição da umidade no interior da madeira são de grande importância na segregação das peças em teores de umidade mais uniformes, de modo a facilitar a secagem tanto no que diz respeito à minimização de defeitos quanto à obtenção de menor variabilidade em torno do teor de umidade médio pretendido. A umidade da madeira na árvore pode variar de 31 a $249 \%$ no cerne e de 40-213\% no alburno (FOREST P...., 1987). Para árvores de Xylopia sericea, o teor de umidade médio encontrado foi de $74 \%$, com acentuada variação no sentido medula-casca e ao longo do tronco, sendo a região interna mais úmida (OLIVEIRA et al., 1990). Em Eucalyptus maculata, a variação encontrada no teor de umidade foi de 50-70\%, no E. citriodora de 46-80\% e no E. grandis de 50-133\% (CARMO, 1996).

A densidade básica da madeira é reconhecida como um dos mais importantes parâmetros para avaliação da sua qualidade, por ser de fácil determinação e estar relacionada às suas demais características (SHIMOYAMA e BARRICHELLO, 1991). Na caracterização da madeira, a determinação de sua densidade e principalmente de sua variação dentro da árvore, tanto na direção radial, da medula para a casca, quanto no sentido base-topo, é fundamental como subsídio ao entendimento de sua qualidade.
Madeira mais homogênea, no que diz respeito à sua densidade no interior do tronco, sem dúvida nenhuma poderá se comportar melhor nas operações de processamento e refletir maior uniformidade nas demais propriedades tecnológicas. As peças de madeira com menor variação de densidade são adequadas para utilizações que exigem material homogêneo e com menor variabilidade nas propriedades fisico-mecânicas.

Diferentes níveis de variação da densidade da madeira ocorrem, da mesma forma, no tronco de outras espécies de folhosas e coníferas, como Hampea appendiculata e Ochroma pyramidale (WIEMANN e WILLIAMSON, 1988), Xylopia sericea (OLIVEIRA et al., 1990), Araucaria cunninghamii, Pinus caribaea e Pinus oocarpa (OLIVEIRA e DELLA LUCIA, 1994).

Pela importância da determinação da umidade e da densidade da madeira, o presente trabalho teve como objetivo avaliar as variações do teor de umidade e da densidade básica na direção radial e longitudinal do tronco de árvores recém-abatidas de sete espécies de eucalipto.

\section{MATERIAL E MÉTODOS}

No presente estudo, utilizou-se a madeira de Eucalyptus citriodora, E. tereticornis, E. paniculata, E. pilularis, E. cloeziana, E. urophylla e E. grandis, com idade aproximada de 16 anos, bem como cinco árvores de cada espécie mencionada de uma plantação da Estação Experimental de Anhembi, SP, do Departamento de Ciências Florestais da ESALQ/USP. A caracterização das parcelas quanto a época de plantio, espaçamento e procedência das sementes está sumariada no Quadro 1.

Quadro 1-Espécies e procedências de eucaliptos, idades e espaçamentos dos plantios experimentais Table 1 -Eucalypt species and provenances, age and spacing of the experimentals plots

\begin{tabular}{llc}
\hline ESPÉCIE & \multicolumn{1}{c}{ PROCEDÊNCIA/PROGÊNIE } & $\begin{array}{c}\text { ÉPOCA DE } \\
\text { PLANTIO }\end{array}$ \\
\hline E. citriodora & 34 progênies da Austrá-lia, 5 procedências da CAF-MG. & $22 / 05 / 80$ \\
E. tereticornis & População base de multi-procedências & $22 / 05 / 80$ \\
E. paniculata & Sementes - Horto de Rio Claro-SP & 3 X 2 \\
E. pilularis & 22 progênies da Austrália, procedências de Zimbabue, & 3 X 2 \\
& e 2 procedên cias de Mogi Guaçu-SP & $30 / 03 / 79$ \\
E. cloeziana & 16 matrizes de diversas procedências da Austrália 2 & 32 \\
E. urophylla & Procedências de Timor e Indonésia 3 \\
E. grandis & 25 matrizes de Atherton- Austrália & $22 / 05 / 80$ \\
\hline
\end{tabular}

R. Árvore, Viçosa-MG, v.29, n.1, p.115-127, 2005 
Foram amostradas árvores internas de parcelas de área aproximada de 1 ha para cada espécie estudada. As árvores escolhidas ao acaso no interior das parcelas estiveram dentro de um intervalo de confiança de 5\% para cima ou para baixo em relação ao DAP médio.

Após a derrubada das árvores foram retirados discos de madeira na base, a 25, 50, 75 e 100\% da altura comercial (diâmetro igual a $5 \mathrm{~cm}$ ), os quais foram acondicionados em sacos plásticos para a prevenção da perda de umidade, sendo, em seguida, retiradas amostras radiais de 2,5 $\mathrm{cm}$ de largura.

Para a determinação do teor de umidade pelo método gravimétrico e da densidade básica da madeira, foram cortados corpos-de-prova de 1,0 x 2,5 x $5,0 \mathrm{~cm}$, sendo a última dimensão no sentido longitudinal, a cada 1,0 $\mathrm{cm}$ a partir da medula em direção à casca, nas cinco diferentes alturas do tronco das árvores.

Na determinação do volume das amostras de madeira, na condição verde, utilizou-se o método da balança hidrostática (ABNT, 1940), com a substituição do mercúrio pela água.

\section{RESULTADOS E DISCUSSÃO}

\subsection{Teor de umidade da madeira}

O teor de umidade da madeira tem seus valores apresentados no Quadro 2.
Os valores médios do teor de umidade de cada espécie de eucalipto variaram de 62 (Eucalyptus citriodora, densidade básica igual a $\left.0,73 \mathrm{~g} / \mathrm{cm}^{3}\right)$ a $102 \%$ (Eucalyptus grandis, densidade básica igual a 0,49 $\mathrm{g} / \mathrm{cm}^{3}$ ). Nas madeiras mais densas, os teores de umidade foram mais baixos, como em E. citriodora, E. paniculata e E. cloeziana, com valores de densidade básica de 0,$73 ; 0,72$; e $0,72 \mathrm{~g} / \mathrm{cm}^{3}$, respectivamente. Valores intermediários de teor de umidade foram encontrados no E. tereticornis e em E. pilularis, de densidade básica de 0,66 e $0,60 \mathrm{~g} / \mathrm{cm}^{3}$, respectivamente, e mais elevados em E. urophylla e E. grandis, cujos valores de densidade básica são de 0,54 e $0,49 \mathrm{~g} / \mathrm{cm}^{3}$, respectivamente. Os mais baixos coeficientes de variação foram obtidos para a madeira de E. paniculata $(15,8 \%)$ e E. citriodora $(18,1 \%)$, que associados aos desvios-padrão indicam menor variabilidade e maior homogeneidade quanto à distribuição de umidade. Ao contrário, nas demais espécies os coeficientes de variação estão acima de $20 \%$, evidenciando, portanto, alta variabilidade dos dados. Observou-se, também, elevada variabilidade da umidade da madeira entre e dentro das árvores de uma mesma espécie, com os valores mais baixos de coeficiente de variação e desvio-padrão para o $E$. citriodora e E. paniculata. A variabilidade para esse parâmetro é também encontrada ao longo da altura e no sentido radial do tronco das árvores (Quadro 3).

Quadro 2 - Teores de umidade médio (\%) por árvore e geral para a madeira de sete espécies de Eucalyptus Table 2 - Average of moisture content (\%) by tree and in general in the wood from seven eucalypt species

\begin{tabular}{|c|c|c|c|c|c|c|c|}
\hline \multirow[t]{2}{*}{ ESPÉCIE } & \multirow[t]{2}{*}{$\mathrm{N}^{1}$} & \multicolumn{6}{|c|}{ NÚMERO DA ÁRVORE } \\
\hline & & 1 & 2 & 3 & 4 & 5 & Geral \\
\hline \multirow[t]{3}{*}{$\overline{\text { E. citriodora }}$} & 191 & 59,6 & 63,6 & 59,1 & 72,6 & 57,7 & 62,6 \\
\hline & & $9,2^{2}$ & 12,9 & 8,7 & 10,3 & 7,9 & 11,3 \\
\hline & & $15,4^{3}$ & 20,3 & 14,7 & 14,2 & 13,6 & 18,1 \\
\hline \multirow[t]{3}{*}{ E. Tereticornis } & 186 & 82,6 & 79,6 & 85,1 & 65,5 & 70,1 & 76,5 \\
\hline & & 22,3 & 14,0 & 23,0 & 15,2 & 17,9 & 20,2 \\
\hline & & 27,0 & 17,6 & 27,1 & 23,3 & 25,5 & 26,4 \\
\hline \multirow{3}{*}{ E. paniculata } & 176 & 64,1 & 59,6 & 64,7 & 63,1 & 57,9 & 62,0 \\
\hline & & 14,9 & 8,5 & 6,7 & 7,4 & 6,6 & 9,8 \\
\hline & & 23,2 & 14,3 & 10,3 & 11,8 & 11,4 & 15,8 \\
\hline \multirow{3}{*}{ E. pilularis } & 200 & 84,5 & 78,4 & 76,0 & 88,8 & 103,3 & 86,3 \\
\hline & & 22,0 & 18,0 & 16,6 & 18,5 & 31,0 & 23,9 \\
\hline & & 25,9 & 23,0 & 21,9 & 20,9 & 30,0 & 27,7 \\
\hline \multirow[t]{3}{*}{ E. cloeziana } & 189 & 63,3 & 62,7 & 58,5 & 70,0 & 64,8 & 63,9 \\
\hline & & 14,7 & 13,7 & 13,2 & 19,7 & 17,2 & 16,2 \\
\hline & & 23,2 & 21,9 & 22,5 & 28,2 & 26,5 & 25,3 \\
\hline \multirow[t]{3}{*}{ E. urophylla } & 213 & 102,4 & 97,4 & 92,5 & 81,7 & 107,5 & 96,2 \\
\hline & & 25,3 & 24,3 & 18,0 & 23,1 & 27,4 & 25,2 \\
\hline & & 24,8 & 24,9 & 19,5 & 28,3 & 25,5 & 26,2 \\
\hline \multirow{3}{*}{ E. grandis } & 249 & 106,9 & 92,0 & 107,5 & 105,7 & 98,6 & 102,3 \\
\hline & & 24,3 & 26,6 & 20,7 & 27,7 & 28,0 & 26,0 \\
\hline & & 22,7 & 28,8 & 19,2 & 26,2 & 28,4 & 25,4 \\
\hline
\end{tabular}

(1) - número de observações, (2)- valores de desvio-padrão (\%) e (3) - valores de coeficiente de variação (\%). 
Quadro3-Sumário das análises de variância do teor de umidade da madeira de sete espécies de Eucalyptus

Table 3 -Summary of the analysis of variance of moisture content of the wood from seven eucalypt species

\begin{tabular}{lccc}
\hline \multirow{2}{*}{ ESPÉCIE } & \multicolumn{3}{c}{ FONTE DE VARIAÇÃO } \\
\cline { 2 - 4 } & Árvore(4) & Altura (4) & Raio no DAP \\
\hline E. citriodora & $* *$ & $* *$ & $*$ \\
E. tereticornis & $* *$ & $* *$ & $* *(12)$ \\
E. paniculata & $*$ & $* *$ & $* *(10)$ \\
E. pilularis & $* *$ & $*$ & $* *(15)$ \\
E. cloeziana & $*$ & $* *$ & $* *(12)$ \\
E. urophylla & $* *$ & $* *$ & $* *(13)$ \\
E. grandis & $* *$ & $* *$ & $* *(18)$ \\
\hline
\end{tabular}

1 - graus de liberdade.

* - altamente significativo a $1 \%$ de probabilidade.

** - significativo a $5 \%$ de probabilidade.
A variação do teor de umidade da madeira ao longo do tronco e na radial - na altura do DAP - entre árvores foi significativa em todas as espécies, à exceção do E. paniculata e E. cloeziana (variação entre árvores), E. pilularis (ao longo do tronco) e E. citriodora (variação radial), com nível de significância de $1 \%$. Para efeito de comparação, as madeiras de E. citriodora, E. pilularis, E. cloeziana e E. grandis, de árvores de 21 anos estudadas por Carmo (1996), apresentaram valores de teor de umidade máximos de 52, 58, 54 e $70 \%$, respectivamente. A variação do teor de umidade médio da madeira das cinco árvores das espécies de eucalipto na direção radial, nas cinco posições ao longo do tronco, é apresentada na Figura 1.
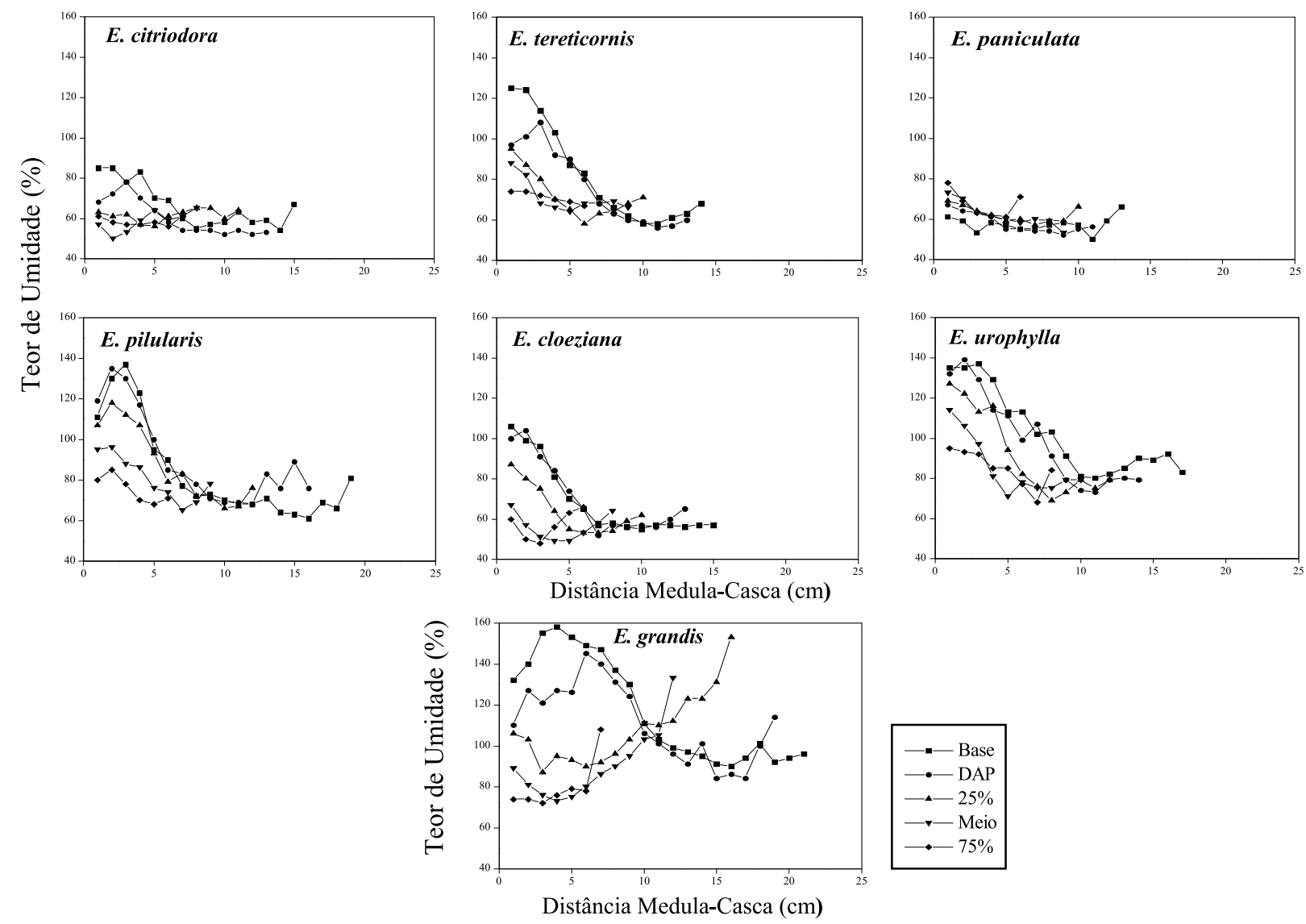

Figura 1 - Variação do teor de umidade da madeira na direção medula-casca, ao longo do tronco para sete espécies de Eucalyptus.

Figure 1 -Variation of the moisture content of the wood in the pith-bark and bottom-top direction from seven eucalypt species.

R. Árvore, Viçosa-MG, v.29, n.1, p.115-127, 2005 
Na Figura 1, mostra-se o gradiente de umidade da madeira da medula em direção à casca das árvores, com nítida distinção em função da espécie e posição ao longo do tronco. Destaca-se a distribuição mais uniforme do teor de umidade na madeira de E. citriodora e E. paniculata, reflexo de uma estrutura anatômica mais homogênea e da sua constituição química, com o diferencial de $20 \%$ de umidade da região mais interna para a mais externa do tronco. Em E. urophylla e $E$. grandis, os valores dessa diferença de umidade são de $80 \%$ e em E. tereticornis e E. pilularis, de $60 \%$. À exceção do E. grandis, as demais espécies apresentaram tendência de queda do teor de umidade da madeira a partir da medula, com posterior estabilização e ligeiro acréscimo na região periférica do alburno. No E. grandis, esse modelo é mantido na base e no DAP e inverso a partir da primeira quarta parte do tronco das árvores. Pode-se entender a variação de umidade da madeira dessas espécies pela avaliação da espessura do alburno na base do tronco, onde citado por Oliveira et al. (1999), em E. citriodora, E. paniculata e E. cloeziana, o alburno foi de 3,8, 4,7 e 2,2 cm de espessura, respectivamente, com uma flutuação muito baixa do teor de umidade indicando a formação de madeira mais uniforme. Em E. tereticornis e E. urophylla, na região basal do tronco, conforme Oliveira et al. (1999), o alburno foi de 2,4 e 4,5 cm, respectivamente, verificando-se tendência de queda no gradiente de umidade; em $E$. pilularis com 1,9 cm de alburno, houve leve tendência de acréscimo do teor de umidade nos $4 \mathrm{~cm}$ finais do raio; em E. grandis com 3,4 cm de alburno, segundo Oliveira et al. (1999), o modelo das outras espécies não foi detectado. Nesse sentido, Carmo (1996) verificou que os teores de umidade mais elevados da madeira ocorrem na base do tronco das árvores e próximo da medula, com diminuição nos sentidos longitudinal e radial, com a ressalva de que o teor de água indica valor mínimo no cerne mais externo, elevando-se novamente no alburno em árvores de E. grandis. Shupe et al. (1995) afirmaram que as maiores diferenças entre a secagem das madeiras de folhosas e coníferas americanas relacionam-se com as diferenças do teor de umidade do cerne e do alburno; as coníferas possuem o cerne com teor de umidade muito inferior ao do alburno, ao contrário das folhosas, com alto teor de umidade no cerne, embora essa relação seja altamente variável. Com relação às árvores de E. camaldulensis, com 1820 anos de idade, na região basal do tronco reportouse um alto teor de umidade na região da medula (103\%), que se reduz no cerne mediano $(80 \%)$ e volta a aumentar no alburno (92\%) (HILLIS, 1978).

A Figura 2 ilustra a variação do teor de umidade da madeira ao longo da altura do tronco, com tendência de a base apresentar maior teor de umidade, diminuindo até a metade da altura (à exceção de E. tereticornis e E. paniculata) e com novo acréscimo a partir do terço superior e no topo, com teor de umidade próximo ao da base da árvore.

E. grandis possui as maiores variações; com $120 \%$ de umidade na base, reduzindo para 80 a $75 \%$ de altura, elevando-se novamente para $90 \%$ no topo ou altura comercial do tronco. E. urophylla apresentou variação de teor de umidade da madeira ligeiramente maior no topo da árvore, com modelo de variação semelhante ao de E. grandis. No E. tereticornis, o teor de umidade da madeira decresceu da base até um quarto da altura, atingindo o valor mais elevado na extremidade do tronco. No E. paniculata, o teor de umidade mostrou-se crescente a partir da base até o ápice do tronco, com variação de aproximadamente $20 \%$.

A variação do teor de umidade da madeira nas direções radial e longitudinal no tronco das árvores é negativamente correlacionada com a sua densidade; madeiras com maior densidade possuem menor volume de vazios internos e, portanto, menor espaço para preenchimento com água livre ou de capilaridade.

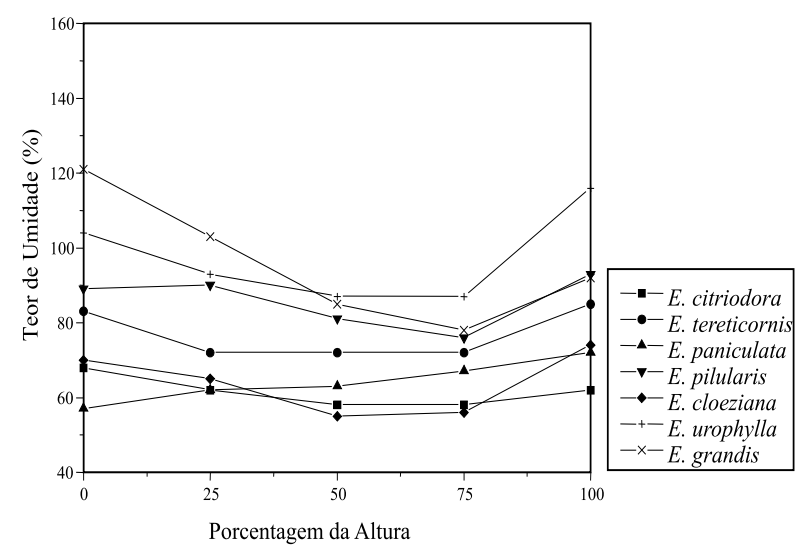

Figura 2 - Variação do teor de umidade da madeira, ao longo do tronco, de sete espécies de Eucalyptus.

Figure 2-Variation of the moisture content of the wood in the bottom-top direction of seven eucalypt species.

R. Árvore, Viçosa-MG, v.29, n.1, p.115-127, 2005 
Os resultados relativos à distribuição de umidade evidenciaram espécies de eucaliptos com madeira de estrutura anatômica homogênea como E. paniculata e E. citriodora e que poderá refletir melhor estabilidade em relação ao $E$. grandis, por exemplo. A umidade da madeira tem, também, implicação com o seu processamento, uma vez que na secagem as peças com maiores gradientes de umidade tendem a apresentar defeitos, como o fendilhamento e o empenamento. As deformações das peças de madeira decorrentes das contrações de secagem, nas espécies com elevado gradiente de umidade, podem ser minimizadas pela separação das tábuas em classes de umidade. A adoção de técnicas especiais de serraria, permitindo o desdobro de peças com variação mínima de teor de umidade, poderá, da mesma forma, contribuir para o rendimento e utilização final da madeira.

\subsection{Densidade básica da madeira}

Os valores médios de densidade básica por árvore e geral das espécies de eucalipto são apresentados na Tabela 4.

Destacam-se E. citriodora, E. cloeziana e E. paniculata, cujas madeiras estão incluídas na mesma faixa de densidade e com baixos valores de coeficientes de variação. As demais espécies diferiram estatisticamente, com E. grandis apresentando madeira com menor valor de densidade e variação significativa entre árvores, confirmada por vários autores (TOMAZELLO FILHO, 1994), no caso particular do gênero Eucalyptus e da madeira de modo geral (KOLLMAN e COTÊ, 1968; PANSHIN e DE ZEEUW, 1980). Na Austrália ocorrem variações importantes na densidade da madeira de árvores de uma mesma espécie, como E. pilularis, com variações de 0,60$0,80 \mathrm{~g} / \mathrm{cm}^{3}$ (HILLIS eBROWN, 1978). Árvores desta espécie, aos 22 anos, em Viçosa, MG produziram madeira de densidade básica média de 0,64 g/ $\mathrm{cm}^{3}$ (LIMA, 1996), superior a 0,60 $\mathrm{g} / \mathrm{cm}^{3}$, das árvores de 16 anos (Tabela 4). Árvores de E. pilularis e E. grandis, aos 10 anos de idade, em Salesópolis, SP, exibiram valores de densidade básica da madeira de $0,50 \mathrm{e} 0,40 \mathrm{~g} / \mathrm{cm}^{3}$, respectivamente (TOMAZELLOFILHO, 1985), sendo as diferenças atribuídas, da mesma forma, à idade das árvores, entre outros fatores. $\mathrm{O}$ aumento da densidade básica da madeira de árvores de eucalipto varia com a idade, com tendência de estabilização após a formação da madeira adulta, havendo, portanto, necessidade de se determinar a idade das árvores para fins de comparação, bem como as condições do sítio (RIBEIRO e ZANI FILHO, 1993). Em árvores de E. grandis, foi confirmado o aumento sistemático da densidade da madeira com a idade das árvores (VITALet al., 1987). A variabilidade da densidade da madeira de árvores de espécies de eucaliptoé confirmada pela análise de variância das fontes de variação em árvore, altura e direção radial no DAP (Quadro 5).

Quadro 4 -Densidade básica média, em $\mathrm{g} / \mathrm{cm}^{3}$, da madeira de sete espécies de Eucalyptus Table 4-Average specific gravity in $\mathrm{g} / \mathrm{cm}^{3}$ in the wood of seven eucalypt species

\begin{tabular}{|c|c|c|c|c|c|c|c|}
\hline \multirow[t]{2}{*}{ ESPÉCIE } & \multirow[t]{2}{*}{$\mathrm{N}^{1}$} & \multicolumn{6}{|c|}{ NÚMERO DA ÁRVORE } \\
\hline & & 1 & 2 & 3 & 4 & 5 & Geral \\
\hline \multirow[t]{3}{*}{ E. citriodora } & 249 & $0,73 b^{1}$ & $0,74 \mathrm{ab}$ & $0,73 b$ & $0,67 \mathrm{c}$ & $0,77 a$ & $0,73 \mathrm{~A}^{2}$ \\
\hline & & $0,08^{3}$ & 0,06 & 0,05 & 0,05 & 0,06 & 0,07 \\
\hline & & $10,5^{4}$ & 8,7 & 7,2 & 7,4 & 7,9 & 9,3 \\
\hline \multirow[t]{3}{*}{ E. cloeziana } & 249 & $0,73 \mathrm{~b}$ & $0,68 \mathrm{~b}$ & $0,78 \mathrm{a}$ & $0,70 b$ & $0,70 \mathrm{~b}$ & $0,72 \mathrm{~A}$ \\
\hline & & 0,08 & 0,08 & 0,07 & 0,08 & 0,08 & 0,09 \\
\hline & & 10,4 & 12,3 & 9,1 & 11,8 & 12,2 & 11,9 \\
\hline \multirow[t]{3}{*}{ E. paniculata } & 226 & $0,68 \mathrm{c}$ & $0,72 \mathrm{~b}$ & $0,73 b$ & $0,71 b$ & $0,76 \mathrm{a}$ & $0,72 \mathrm{~A}$ \\
\hline & & 0,06 & 0,05 & 0,04 & 0,03 & 0,04 & 0,05 \\
\hline & & 8,2 & 6,8 & 5,3 & 5,0 & 5,1 & 7,1 \\
\hline \multirow{3}{*}{ E. tereticornis } & 247 & $0,65 b c$ & $0,65 b c$ & $0,62 c$ & $0,71 \mathrm{a}$ & $0,68 \mathrm{ab}$ & $0,66 \mathrm{~B}$ \\
\hline & & 0,11 & 0,07 & 0,09 & 0,08 & 0,10 & 0,09 \\
\hline & & 16,4 & 10,6 & 15,1 & 11,3 & 14,3 & 14,3 \\
\hline \multirow[t]{3}{*}{ E. pilularis } & 263 & $0,58 \mathrm{~cd}$ & $0,64 \mathrm{ab}$ & $0,65 \mathrm{a}$ & $0,59 \mathrm{bc}$ & $0,53 \mathrm{~d}$ & $0,60 \mathrm{C}$ \\
\hline & & 0,10 & 0,08 & 0,07 & 0,07 & 0,10 & 0,10 \\
\hline & & 17,5 & 12,1 & 11,4 & 12,1 & 18,9 & 16,1 \\
\hline \multirow[t]{3}{*}{ E. urophylla } & 280 & $0,53 \mathrm{~b}$ & $0,55 \mathrm{ab}$ & $0,54 b$ & $0,59 a$ & $0,50 \mathrm{~b}$ & $0,54 \mathrm{D}$ \\
\hline & & 0,09 & 0,10 & 0,08 & 0,09 & 0,10 & 0,10 \\
\hline & & 17,9 & 18,0 & 15,6 & 15,4 & 19,0 & 17,8 \\
\hline \multirow{3}{*}{ E. grandis } & 333 & $0,46 \mathrm{~b}$ & $0,53 \mathrm{a}$ & $0,48 b$ & $0,48 \mathrm{~b}$ & $0,49 \mathrm{~b}$ & $0,49 \mathrm{E}$ \\
\hline & & 0,07 & 0,08 & 0,06 & 0,08 & 0,08 & 0,08 \\
\hline & & 15,0 & 15,6 & 13,0 & 15,8 & 16,4 & 15,9 \\
\hline
\end{tabular}

(*) - número de observações, (1) - médias de densidade básica seguidas de mesma letra minúscula na horizontal não diferem estatisticamente pelo teste de Tukey a 5\%. (2) - médias de densidade básica seguidas de mesma letra maiúscula na vertical não diferem estatisticamente pelo teste de Tukey a $5 \%,(3)$ - valores de desvio-padrão $\left(\mathrm{g} / \mathrm{cm}^{3}\right)$ e (4) - valores de coeficiente de variação (\%).

R. Árvore, Viçosa-MG, v.29, n.1, p.115-127, 2005 
Quadro 5-Sumário das análises de variância da densidade básica da madeira de sete espécies de Eucalyptus

Table 5-Summary of the analysis of variance of specific gravity of the wood of seven eucalypt species

\begin{tabular}{lccr}
\hline \multirow{2}{*}{ ESPÉCIE } & \multicolumn{3}{c}{ FONTE DE VARIAÇÃO } \\
\cline { 2 - 4 } & Árvore(4) & Altura (4) & Raio no DAP \\
\hline E. citriodora & $* *$ & $\mathrm{n} . \mathrm{s}^{2}$ & $* *(12)$ \\
E. tereticornis & $* *$ & $*$ & $* *(12)$ \\
E. paniculata & $* *$ & $*$ & $* *(10)$ \\
E. pilularis & $* *$ & $* *$ & $* *(15)$ \\
E. cloeziana & $* *$ & $* *$ & $* *(12)$ \\
E. urophylla & $* *$ & $*$ & $* *(13)$ \\
E. grandis & $* *$ & $* *$ & $* *(18)$ \\
\hline 1 - graus de liberdade. & & \\
2 - não-significativo. & & \\
$*$ - significativo a 1 \% de probabilidade. & \\
$* *$ - significativo a 5 $\%$ de probabilidade.
\end{tabular}

As variações significativas entre árvores de uma mesma espécie (Quadro 5) confirmam o teste de média (Quadro 4). Com relação à altura, o elevado nível de significância indica alta variação em E. tereticornis, E. paniculata e E. urophylla. Ocorreu variação estatisticamente não-significativa na densidade básica da madeira ao longo do tronco de E. citriodora, confirmando estudo anterior não publicado de árvores de 55 anos, em Rio Claro, SP, apontando valores de densidade básica da madeira de 0,83;0,79; e 0,79 g/ $\mathrm{cm}^{3}$ na região da base, meio e topo do tronco, respectivamente. Na direção radial, a variação da densidade básica da madeira foi significativa, confirmando resultados obtidos por diversos autores (WISKAS, 1984; TOMAZELLO FILHO, 1985; SHIMOYAMA E BARRICHELLO, 1991; LIMA, 1996; CARMO, 1996).

Os resultados da variação radial da densidade básica da madeira das espécies de eucalipto, nas posições ao longo do tronco, são apresentados na Figura 3. Os valores correspondentes às amostras das alturas máximas (topo das árvores) não foram apresentados.
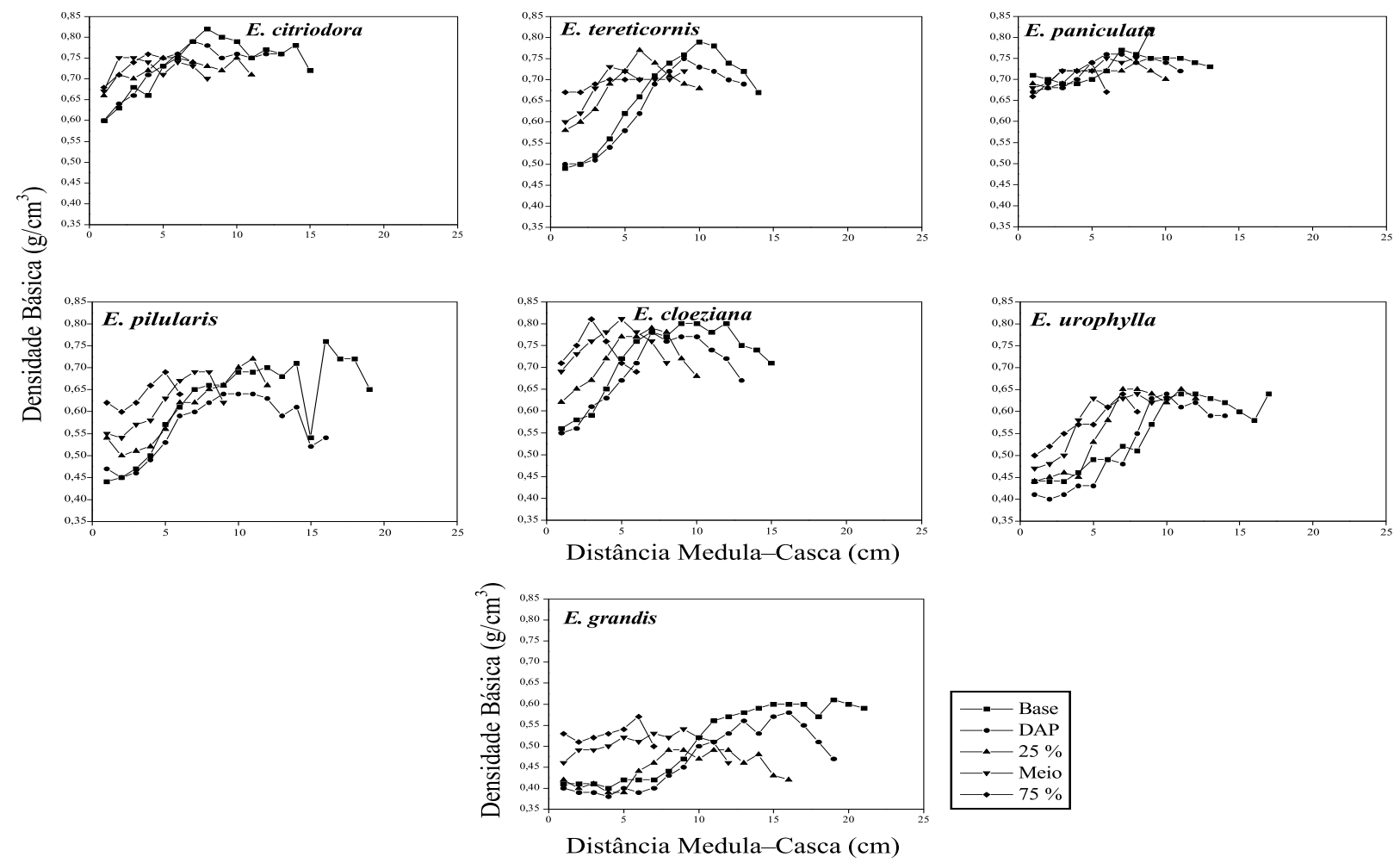

Figura 3 - Variação da densidade básica da madeira na direção medula-casca e ao longo do tronco de sete espécies de Eucalyptus.

Figure 3 -Variation of the specific gravity of the wood in the pith-bark and bottom-top direction from seven eucalypt species. 
Verificou-se uma tendência geral das espécies com a densidade básica da madeira de atingir valores mais baixos na região da medula, aumentando até próximo da região do alburno e seguindo-se, então, uma redução do seu valor (Figura 3). Apesar da tendência geral, cada espécie mostra um perfil diferenciado de variação da densidade da madeira nas diversas alturas do tronco. No DAP das árvores de E. citriodora ocorreu menor variação dos valores de densidade da madeira, indicando a formação de madeira mais uniforme e apropriada para uma ampla gama de utilização. No E. tereticornis, o máximo valor da densidade básica da madeira ocorreu na região externa do cerne, com uma nítida tendência de queda no alburno. Nessa espécie, a menor variabilidade da densidade básica da madeira no alburno foi confirmada na altura de $75 \%$ do tronco, com a porcentagem de alburno ultrapassando $90 \%$, ressaltando-se que em árvores com DAP a partir de 18,0 cm haverá a formação de madeira de menor variação de densidade básica e, portanto, mais homogênea. As menores variações radiais de densidade básica da madeira de E. paniculata parecem relacionadas com a alta porcentagem de alburno, atingindo $63 \%$ na base do tronco, com a ressalva de que a madeira mais uniforme começa a ser formada em árvores com DAP de $15 \mathrm{~cm}$. A variação da densidade básica da madeira de E. pilularis foi, de modo geral, semelhante à de E. citriodora e E. tereticornis, verificando-se em praticamente todas as posições do tronco uma sensível queda no início do alburno, seguindo modelo de variação semelhante ao das demais espécies. E. cloeziana mostrou variação semelhante ao $E$. citriodora e E. tereticornis, com o início da estabilização dos valores de densidade básica da madeira na região do alburno, em que a partir do DAP de $14 \mathrm{~cm}$ as árvores iniciam a produção de madeira com menor variabilidade radial. Em E. urophylla, a variação da densidade básica da madeira indicou perfil definido e semelhante ao das demais espécies. Em árvores com tronco de diâmetro superior a $20 \mathrm{~cm}$, inicia-se a formação de madeira mais uniforme. No E. grandis foi maior a variação radial da densidade da madeira, exceto nas posições basais do tronco das árvores, quando a variação foi semelhante à das demais espécies. As variações na densidade básica da madeira (Figura 3) são semelhantes às citadas na literatura. Rezende e Ferraz (1985) afirmaram que no gênero Eucalyptus o modelo de variação mais comum é caracterizado pelo aumento da densidade básica da madeira no sentido medula-casca. Tomazello Filho(1985), estudando árvores de 10 anos, em Salesópolis, SP, concluiu, com realação a E. grandis e E. pilularis, que a densidade básica aumenta no sentido radial. Lima (1996) verificou em árvores de 22 anos que a densidade da madeira tende a aumentar da medula para o cerne periférico, com redução na região do alburno. Carmo (1996), em árvores de 21 anos de E. grandis, E. citriodora e E. cloeziana, verificou o mesmo modelo de variação. Em duas árvores de E. citriodora de 52 anos e DAP de $26 \mathrm{~cm}$, de Rio Claro, SP, em estudo não publicado, verificou-se que a variabilidade radial da densidade da madeira foi muito baixa na base, meio e topo do tronco, em razão do crescimento lento. Hillis e Brown (1978) relataram na região basal das árvores de E. grandis, aos 16-20 anos, valores de densidade básica da madeira 1,25 vez maior em relação à madeira de 1-5 anos; em E. pilularis, a densidade básica da madeira aumenta com a idade das árvores entre 4-10 anos, e entre 130 anos o diferencial de densidade básica foi de $40 \%$, com valores de $0,54 \mathrm{~g} / \mathrm{cm}^{3}$, podendo atingir $0,70 \mathrm{~g} /$ $\mathrm{cm}^{3}$ em árvores mais velhas.

A variação radial da densidade básica da madeira no DAP das árvores evidencia a diferença entre as espécies (Figura 4). No E. citriodora, à exceção da árvore 4, os valores de densidade da madeira exibem variações pequenas na mesma posição radial, com menor variação desse parâmetro a 8 e $6 \mathrm{~cm}$ nas árvores 4 e 5 , respectivamente, e entre esses intervalos nas demais. Isso confirma que, nas árvores dessa espécie, a partir de $15 \mathrm{~cm}$ de DAP (Figura 3) inicia-se a formação de madeira com propriedades mais estáveis. No $E$. tereticornis, a menor variação radial dos valores de densidade da madeira encontra-se em um raio de 9,0 cm (árvore 4) a 11,0 cm (árvores 1, 2, 3 e 5), com os limites da formação da madeira mais homogênea nas árvores com valores de 18,0-22,0 cm de DAP (Figura 3). À exceção do E. paniculata, as demais espécies apontaram madeira com menor variação de densidade básica próxima ao alburno. No E. paniculata, a partir de 3-4 cm da medula a densidade básica da madeira tende a aumentar até $6 \mathrm{~cm}$ do raio (árvores 1,3 e 5).

Na Figura 4, demonstram-se a complexidade e a variabilidade do parâmetro densidade radial das árvores de eucalipto. Na variação radial da densidade básica da madeira, na altura do DAP verificaram-se diferenças entre as sete espécies de eucalipto (Figura 5), com variações de $0,40-0,80 \mathrm{~g} / \mathrm{cm}^{3}$. Em E. citriodora, $E$. cloeziana e E. paniculata, a madeira de densidade básica menos variável começou a ser formada, na altura do DAP, nas árvores com 14,0 cm; em E. tereticornis

\section{R. Árvore, Viçosa-MG, v.29, n.1, p.115-127, 2005}


e E. pilularis, acima de $18,0 \mathrm{~cm}$; e em E. urophylla e E. grandis, a partir de 20,0 e 25,0-30,0 cm, respectivamente. No E. grandis, a curva de densidade básica da madeira evidencia que os valores são de
$0,40 \mathrm{~g} / \mathrm{cm}^{3}$, em árvores com raio de $15,0 \mathrm{~cm}$, podendose inferir que árvores com DAP inferior a $30,0 \mathrm{~cm}$ produzirão madeira de propriedades anatomofisicomecânicas mais heterogêneas.
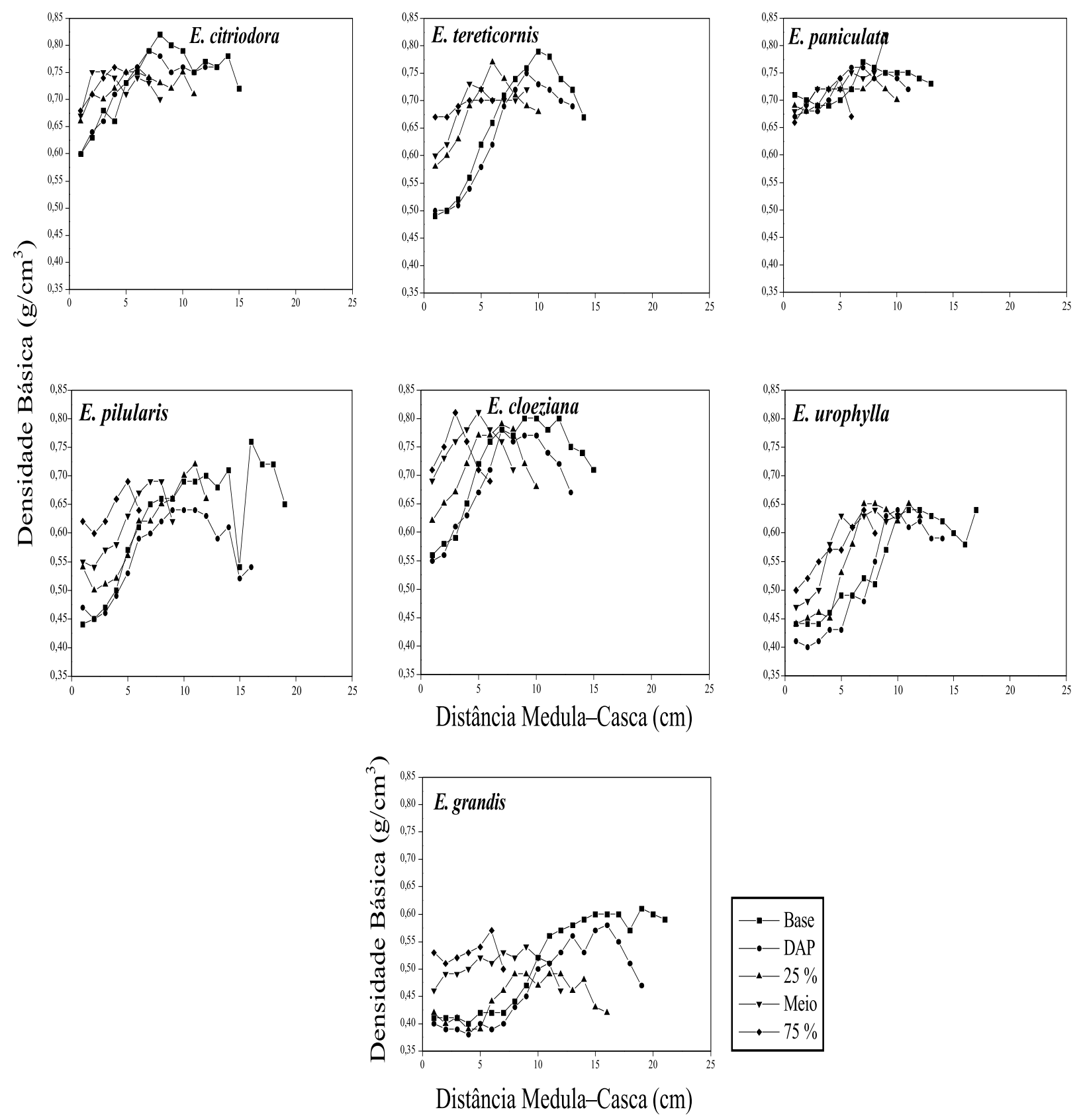

Figura 4 - Variação da densidade básica da madeira na direção radial do DAP de cinco árvores de sete espécies de Eucalyptus. Figure 4-Variation of the specific gravity of the wood in the radial direction at DBH in five trees seven eucalypt species. 


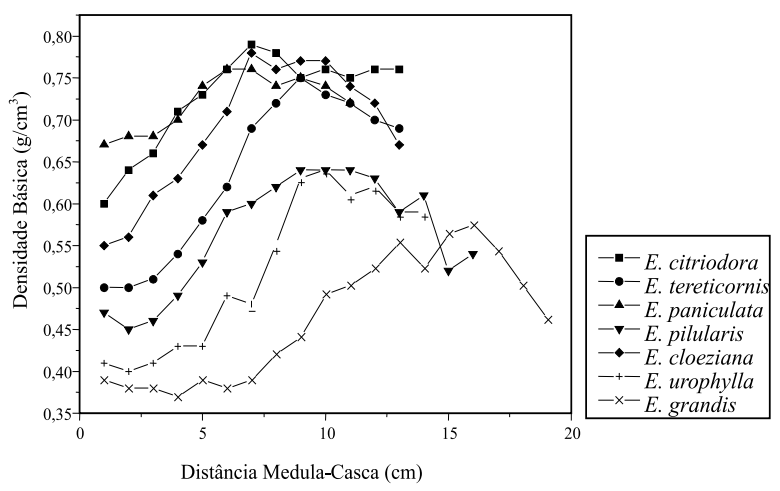

Figura 5 - Variação da densidade básica da madeira na direção medula-casca no DAP de sete espécies de Eucalyptus.

Figure 5 -Variation of the specific gravity of the wood in the pith-bark direction at DBH in seven eucalypt species.

A magnitude da variação da densidade básica da madeira (Quadro 6) indica que as árvores de E. urophylla e E. grandis produzem madeira com 0,54 e $0,49 \mathrm{~g} / \mathrm{cm}^{3}$, respectivamente, com amplitudes elevadas de $0,35 \mathrm{e}$ $0,28 \mathrm{~g} / \mathrm{cm}^{3}$. Em árvores adultas de E. globulus, o valor médio da amplitude de variação para densidade básica da madeira foi de $0,40 \mathrm{~g} / \mathrm{cm}^{3}$ (CARVALHO, 1962); em árvores de 10 anos de E. grandis e E. pilularis, com madeira de densidade básica de 0,38 e $0,46 \mathrm{~g} / \mathrm{cm}^{3}$, respectivamente, esse valor foi de $0,13 \mathrm{~g} / \mathrm{cm}^{3}$ (TOMAZELLO FILHO, 1985). A razão da amplitude de variação/densidade básica da madeira demonstra os seguintes valores: $E$. urophylla $(0,65)>$ E. grandis $(0,57)>$ E. pilularis $(0,53)>E$. tereticornis $(0,48)>$ E. cloeziana $(0,43)>E$. citriodora $(0,35)>E$. paniculata $(0,28)$. Dessa forma, destacaram-se como madeira mais homogênea
E. citriodora e E. paniculata e as mais heterogêneas, E. urophylla e E. grandis, com as demais espécies em níveis intermediários, porém elevados em relação às outras espécies de folhosas, como a pimenteira (Xylopia sericea), com densidade básica de $0,53 \mathrm{~g} /$ $\mathrm{cm}^{3}$ e amplitude de variação de $0,08 \mathrm{~g} / \mathrm{cm}^{3}$. Essa espécie produz madeira de propriedades mais uniformes e variações pequenas nos sentidos radial e longitudinal do tronco (OLIVEIRA, 1988).

A variação longitudinal da densidade básica da madeira de eucalipto (Figura 6), apesar de menor do que a radial, foi altamente significativa (Quadro 5) em E. tereticornis, E. paniculata e E. urophylla, significativa em E. pilularis, E. cloeziana e E. grandis e nãosignificativa em E. citriodora.

À exceção do E. grandis, com aumento de $25 \%$ na densidade básica da madeira, nas demais espécies houve redução no sentido base-topo das árvores, embora mínima em E. citriodora. Em árvores de E. grandis, a densidade da madeira de $0,50 \mathrm{~g} / \mathrm{cm}^{3}$ na base do tronco decresceu para $0,45 \mathrm{~g} / \mathrm{cm}^{3}$ a um quarto da altura, atingindo $0,55 \mathrm{~g} / \mathrm{cm}^{3}$ no topo. A amplitude de variação longitudinal da densidade básica é menor, sendo de $0,10 \mathrm{~g} / \mathrm{cm}^{3}$ em E. grandis.

A literatura apresenta diversos padrões de variação, como as reportadas por Shimoyama e Barrichello (1991) em E. grandis, com sete anos, com o decréscimo da densidade básica da base para o topo e em E. urophylla, o seu aumento com a altura do tronco; e por Carmo (1996) em E. grandis, E. pilularis e E. cloeziana, com a densidade básica da madeira aumentando no sentido base-topo e em E. citriodora com variações nãosignificativas.

Quadro 6 - Amplitude de variação da densidade básica da madeira em g/cm³ geral e na direção radial, nas diversas posições ao longo do tronco, de sete espécies de Eucalyptus

Table 6 - Amplitude of variation of the specific gravity of the wood in $\mathrm{g} / \mathrm{cm}^{3}$ in general and in the radial direction in severals positions in the trunk of seven eucalypt species

\begin{tabular}{lccccccc}
\hline \multirow{2}{*}{ ESPÉCIE } & GERAL & \multicolumn{5}{c}{ ALTURA } \\
\cline { 3 - 8 } & & Base & DAP & $25 \%$ & Meio & $75 \%$ & Topo \\
\hline E. urophylla & 0,35 & 0,39 & 0,40 & 0,38 & 0,30 & 0,21 & 0,15 \\
E. tereticornis & 0,32 & 0,39 & 0,33 & 0,29 & 0,25 & 0,18 & 0,14 \\
E. pilularis & 0,32 & 0,41 & 0,41 & 0,29 & 0,26 & 0,26 & 0,20 \\
E. cloeziana & 0,31 & 0,36 & 0,35 & 0,29 & 0,22 & 0,22 & 0,23 \\
E. grandis & 0,28 & 0,32 & 0,34 & 0,24 & 0,21 & 0,17 & 0,18 \\
E. citriodora & 0,26 & 0,36 & 0,35 & 0,26 & 0,22 & 0,15 & 0,11 \\
E. paniculata & 0,20 & 0,28 & 0,22 & 0,17 & 0,24 & 0,26 & 0,11 \\
\hline
\end{tabular}

R. Árvore, Viçosa-MG, v.29, n.1, p.115-127, 2005 


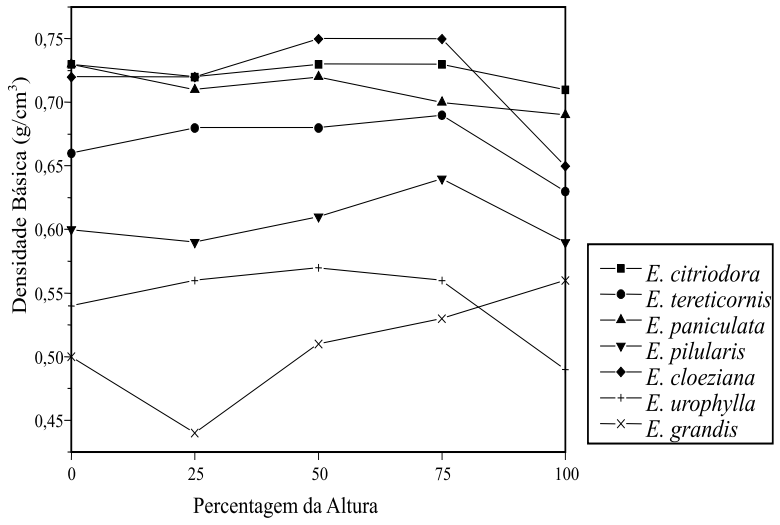

Figura 6 - Variação da densidade básica da madeira ao longo do tronco de sete espécies de Eucalyptus.

Figure 6-Variation of the specific gravity of the wood in the bottom-top direction in seven eucalypt species.

A correlação do teor de umidade verde e densidade básica da madeira foi negativa, apontando valores altos na maioria das espécies (Quadro 7). Com relação à altura das árvores, os coeficientes são altamente significativos, sendo, no entanto, baixos para o teor de umidade. Para a densidade básica da madeira, à exceção do baixo e positivo valor no E. grandis, não houve correlação nas demais espécies. Com respeito ao teor de umidade da madeira versus raio das amostras, apenas no $E$. grandis não houve correlação; o teor de umidade da madeira tende a diminuir na direção medula-casca nas demais espécies. Para densidade básica da madeira, o coeficiente de correlação foi positivo e relacionado com

Quadro 7 - Correlação entre teor de umidade, densidade básica, altura e raio da madeira de sete espécies de Eucalyptus

Table 7 - Correlations between moisture content, specific gravity, height and radius position for the wood of seven eucalypt species

\begin{tabular}{|c|c|c|c|c|c|}
\hline \multirow{3}{*}{ Espécie } & \multicolumn{5}{|c|}{ Coeficiente de Correlação (r) $\quad(p<0$} \\
\hline & \multicolumn{3}{|c|}{$\begin{array}{l}\text { Umidade } \\
\text { versus }\end{array}$} & \multicolumn{2}{|c|}{$\begin{array}{c}\text { Densidade } \\
\text { versus }\end{array}$} \\
\hline & Altura & Raio & Dens & Altura & Raio \\
\hline$E$. & $-\mathrm{C}$ & 4 & & n.s. ${ }^{1}$ & $\overline{0,4}$ \\
\hline & -0 & & & s. & 0,5 \\
\hline & -0 & 0,43 & -0 & s. & 0,4 \\
\hline E. $p$ & -0 & n.s. & -0 & n.s. & 0,5 \\
\hline E. & $-0,89$ & $-0,24$ & -0 & n.s. & 0, \\
\hline & $-0,91$ & $-0,16$ & $-0,52$ & n.s. & 0 , \\
\hline E. grandis & $-0,64$ & $-0,54$ & n.s. & 0,14 & 0 \\
\hline
\end{tabular}

(1) - não significativo ao nível de significância igual a $1 \%$. o aumento da distância da medula-casca, sendo os valores baixos, apesar de altamente significativos (Quadro 7).

Pela análise da variação do teor de umidade e da densidade básica da madeira no DAP (Quadro 8), verificou-se que os coeficientes de correlação aumentam no E. paniculata e E. grandis, principalmente, e são inferiores no E. citriodora, E. tereticornis e E. urophylla. Nessa posição fica evidente o coeficiente de correlação negativo e relativamente alto do teor de umidade da madeira versus distância medula-casca. Quanto à densidade da madeira versus distância medula-casca, a correlação é altamente significativa, crescente e positiva. O coeficiente de correlação entre o teor de umidade verde versus densidade básica de Liriodendron tulipera, de 40 anos, foi de $-0,60$, e a correlação do teor de umidade versus densidade da madeira não foi significativa (SHUPE et al., 1995). A influência da taxa de crescimento das árvores na densidade da madeira é um importante aspecto a ser considerado, sendo no E. grandis e em outras espécies de folhosas de porosidade difusa esse efeito pequeno (PANSHIN e DE ZEEUW, 1980; WISKAS, 1984). Nas espécies de rápido crescimento, um importante parâmetro a ser considerado é a idade de corte das árvores, uma vez que em idades curtas, apesar do significativo diâmetro do tronco, o lenho é formado por grande proporção de madeira juvenil, mostrando heterogeneidade estrutural e comprometendo as suas propriedades físico-mecânicas. Em algumas espécies, como em $E$. pilularis, a madeira adulta é formada somente a partir dos 30 anos (BAMBER e CURTIN, 1974 apud WISKAS, 1984).

Quadro 8 - Correlação entre teor de umidade, densidade básica e raio no DAP para madeira de sete espécies de Eucalyptus

Table 8 -Correlations between moisture content, specific gravity and radius at DBH for the wood of seven eucalypt species

\begin{tabular}{lccc}
\hline \multirow{2}{*}{ Espécie } & \multicolumn{2}{c}{ Coeficiente de Correlação $(\mathrm{r})$} & $(\mathrm{p}<0,01)$ \\
\cline { 2 - 4 } & $\begin{array}{c}\text { Umidade } \\
\text { versus } \\
\end{array}$ & $\begin{array}{c}\text { Umidade } \\
\text { versus } \\
\text { Densidade }\end{array}$ & $\begin{array}{c}\text { Densidade } \\
\text { versus } \\
\text { Raio }\end{array}$ \\
\hline E. citriodora & $-0,68$ & $-0,53$ & 0,59 \\
E. tereticornis & $-0,87$ & $-0,79$ & 0,82 \\
E. paniculata & $-0,92$ & $-0,68$ & 0,54 \\
E. pilularis & $-0,96$ & $-0,62$ & 0,54 \\
E. cloeziana & $-0,92$ & $-0,78$ & 0,67 \\
E. urophylla & $-0,88$ & $-0,70$ & 0,77 \\
E. grandis & $-0,84$ & $-0,56$ & 0,73 \\
\hline
\end{tabular}




\section{CONCLUSÕES}

Quanto à distribuição de umidade na madeira das sete espécies de eucalipto estudadas, concluiu-se que:

(I) O E. paniculata e o E. citriodora foram mais homogêneos e o E. urophylla e o E. grandis, mais heterogêneos.

(II) O E. cloeziana mostrou comportamento mais uniforme de variação da umidade.

(III) À exceção do E. grandis, verificou-se nas demais espécies a queda do gradiente de umidade no sentido medula-casca.

(IV) $\mathrm{O}$ teor de umidade foi mais elevado na base do tronco, diminuindo até por volta da sua metade, aumentando a partir do terço superior, com valores no topo próximos aos da base da árvore.

Quanto à densidade básica da madeira, concluiuse que:

(I) Houve aumento dessa propriedade na direção medula-casca, com cada espécie apresentando um perfil de variação distinto.

(II) Árvores de E. citriodora, E. cloeziana e E. paniculata apresentaram madeira de densidade menos variável a partir de diâmetro de $14,0 \mathrm{~cm}$ sem casca; nas árvores de E. tereticornis e E. pilularis, a partir de 18,0 cm de diâmetro; nas de E. urophylla, 20,0 cm; e nas de E. grandis, a partir de $25,0 \mathrm{~cm}$ de diâmetro.

(III) Quanto à variação ao longo do tronco, à exceção do E. grandis, com acréscimo de $25 \%$, nas demais espécies houve queda da densidade no topo das árvores.

(IV) Quanto à amplitude de variação da densidade básica, verificou-se formação de madeira mais homogênea em E. citriodora e E. paniculata, com E. urophylla e $E$. grandis formando madeira mais heterogênea.

(V) A amplitude de variação dos valores da densidade foi menor ao longo do tronco.

Quanto aos coeficientes de correlação no nível do DAP, estes foram negativos e relativamente elevados para teor de umidade versus distância radial e positivos para a densidade da madeira versus sentido radial. Todos foram significativos no nível de $1 \%$.

R. Árvore, Viçosa-MG, v.29, n.1, p.115-127, 2005

\section{REFERÊNCIAS BIBLIOGRÁFICAS}

ASSOCIAÇÃO BRASILEIRA DE NORMAS TÉCNICAS - ABNT. Ensaios físicos e mecânicos da madeira - MB-26/40. Rio de Janeiro, 1940. 16p.

CARMO, A.P.T. Avaliação de algumas propriedades da madeira de seis espécies de eucalipto. 1996.74f. Dissertação (Mestrado em Ciência Florestal) - Universidade Federal de Viçosa.

\section{CARVAlHO, A. Madeira de}

eucalipto(Eucalyptus globulus Labill.) Estudos e observações. Alcobaça: Direção Geral dos Serviços Florestais e Aqüícolas, 1962. 159p.

FOREST PRODUCTS LABORATORY - FPL. Wood handbook: Wood as an engineering material. Washington: U.S.D.A., 1987. 466p. (Agriculture Handbook, 72).

HILLIS, W.E. Wood quality and utilization. In: HILLIS, W.E.; BROWN, A.G., (Eds). Eucalyptus for wood production. Melbourne: CSIRO, 1978. cap.12.

HILLIS, W.E.; BROWN, A.G. (Eds.) Eucalyptus for wood production. Melbourne: CSIRO, 1978.

KOLlmann, F.F.P.; COTÊ, W.A. Principles of wood science and technology. New York: Springer-Verlag, 1968. v.1.

LIMA, T. G. Variações no sentido radial e longitudinal de algumas propriedades das madeiras de Eucalyptus microcorys F. Muell e Eucalyptus pilularis Sm. 1996. $106 \mathrm{f}$. Dissertação (Mestrado em Ciência Florestal) Universidade Federal de Viçosa, 1996.

OLIVEIRA, J.T.S. Estudo das propriedades físicas e tecnológicas da madeira da pindaíba (Xylopia sericeae St. Hil.). 1988. 106f. Dissertação (Mestrado em Ciência Florestal) - Universidade Federal de Viçosa, Viçosa, 1988.

OLIVEIRA, J.T.S.; DELLA LÚCIA, R.M. Propriedades físicas e mecânicas das madeiras de Araucaria cunninghamii, de Pinus caribaea e de P. oocarpa. Viçosa: SIF, 1994. 7p. (Boletim Técnico, 8).

R. Arvore, Viçosa-MG, v.29, n.1, p.115-127, 2005 
OLIVEIRA, J.T.S. et al. Estudo das propriedades físicas e tecnológicas da madeira da pindaíba (Xylopia sericeae St. Hil.). IV. Densidade, umidade e secagem da madeira. Revista Árvore, v.14, n.2, 139-154, 1990.

OLIVEIRA, J.T.S. et al. Caracterização da madeira de sete espécies de eucaliptos para a construção civil: 1. avaliações dendrométricas das árvores. Revista Scientia Forestalis, n. 56, p. 113$124,1999$.

PANSHIN, A.J.; DE ZEEUW, C. Textbook of wood technology. 4.ed. New York, Mc-GrawHill, 1980.

REZENDE, M.A.; FERRAZ, E.S.B. Densidade anual da madeira de Eucalyptus grandis. IPEF, v.30, p.37-41, 1985.

RIBEIRO, F.A.; ZANI FILHO, J. Variação da densidade básica da madeira em espécies/ procedências de Eucalyptus spp. IPEF, v.46, p.76-85, 1993.

SHIMOYAMA, V.R.S.; BARRICHELLO, L.E.G. Influência de características anatômicas e químicas sobre a densidade básica da madeira de Eucalyptus spp. In: CONGRESSO ANUAL DE CELULOSE E PAPEL, 24., 1991, São Paulo. Anais... São Paulo, ABTCP, 1991. p.178-183.
SHUPE T.F. et al. Difference in moisture content and shrinkage between outerwood, middle-wood, and corewood of two yellow-poplar trees. Forest Products Journal, v.45, n.9, p.85-90, 1995.

TOMAZELLO FILHO, M. Estrutura anatômica da madeira de oito espécies de eucalipto cultivadas no Brasil. IPEF, v.29, p.25-36, 1985.

TOMAZELLO FILHO, M. Formação e caracterização da estrutura anatômica da madeira de Eucalyptus. Piracicaba: ESALQ, 1994. 11p.

TOMAZELLO FILHO, M. Variação radial da densidade básica e da estrutura anatômica da madeira do Eucalyptus saligna e E. grandis. IPEF, v.29, p.37-45, 1985.

VITAL, B.R. et al. Efeito da idade da árvore na densidade da madeira de Eucalyptus grandis cultivado na região do cerrado de Minas Gerais. Brasília: IBDF, 1987. p.49-52. (Boletim Técnico, 8)

WIEMANN, M.C.; WILLIAMSON, G.B. Extreme radial changes in wood specific gravity in some tropical pioneers. Wood and Fiber Science, v.20, n.3, p.344- 49, 1988.

WISKAS, J. The influence of rate of growth on the density and heartwood extratives content of eucalypt species. Wood Science and Technologie, v.18, p.113-120, 1984. 
